\title{
A influênca da escolaridade no uso de suplementos alimentares
}

\author{
He influence of education in the use of dietary supplements
}

\author{
Marina Destéfano Prezotto ${ }^{1, *}$ \\ Mara Cléia Trevisan ${ }^{1}$
}

\section{Resumo}

Objetivo: Comparar o uso de suplementos alimentares entre indivíduos com alta e baixa escolaridade os quais são praticantes de atividade física em academias de Uberaba-MG. Métodos: A amostra foi composta por 660 pessoas, com idade acima de 18 anos de ambos os gêneros. Foi utilizado questionário autoaplicável, padronizado, de múltipla escolha, previamente testado. Para análise dos dados foi utilizada a estatística descritiva e o teste Qui-quadrado, por meio do Software SPSS $17 \mathrm{com}$ p-Valor < 0,05. Resultados: É expressiva a prevalência de uso de suplementos alimentares, $317(48 \%)$, principalmente entre os indivíduos com alta escolaridade 234 (35,5\%). Não houve diferença estatística entre o uso de suplementos nos diferentes níveis de escolaridade, entretanto entre os que utilizam, os com alta escolaridade em sua maioria 61 (9,2\%) fazem uso de 2 suplementos, enquanto os com baixa escolaridade em sua maioria $66(10 \%)$, utilizam apenas 1 tipo de suplemento. Os indivíduos com alta escolaridade consomem preferencialmente mais de um tipo de suplemento $161(50,7 \%)$, sendo os ricos em proteínas $35(11 \%)$, anabolizantes $9(2,8 \%)$ os mais consumidos. Já entre os indivíduos com baixa escolaridade se destacam entre os mais consumidos, os ricos em proteínas $18(5,6 \%)$ e BCAA 4 $(1,2 \%)$. Conclusão: $O$ nível de escolaridade não interfere no uso de suplementos alimentares por praticantes de atividade física em academias, porém o uso de suplementos é expressivo nesta população.

Palavras-chave: suplementos nutricionais, academia, nível de escolaridade.

\begin{abstract}
Objective: Compare the use of dietary supplements among individuals with high and low education who are physically active in Uberaba-MG academies. Methods: The sample consisted of 660 people, aged above 18 years and who were physically active (attend the gym at least twice a week), of both genders. Self-administered questionnaire was used, standardized, multiple choice, previously tested. For data analysis descriptive statistics and Chi-square test was used, using the SPSS Software 17 with p-value <0.05. Results: It was observed that most individuals presents a high school $478(72.4 \%)$, the substantial use of food supplements by the participants $317(48 \%)$, mainly in people with high school $234(35.5 \%)$. There was no statistical difference between the use of supplements in different educational levels. However, among those who use the highly educated mostly $61(9.2 \%)$ make use of two supplements, while the poorly educated mostly $66(10 \%)$, use only one type of supplement. Individuals with high levels of education consume preferably more than one type of supplement $161(50.7 \%)$, and the rich protein $35(11 \%)$, anabolic $9(2.8 \%)$ were the most consumed. Among individuals with low education stand out among the most consumed, rich in protein $18(5.6 \%)$ and BCAA $4(1.2 \%)$. Conclusion: The overuse of supplements in both levels of education, points out the need to warn this population about the risks and the need for greater supervision.
\end{abstract}

Keywords: dietary supplements, gym, level of education.

\section{Afiliação dos autores}

${ }^{1}$ Universidade Federal do Triângulo Mineiro, Uberaba, Minas Gerais, Brasil.

\section{${ }^{*}$ Autor correspondente}

Rua Duque de Caxias, 409, apto 202, Bairro São Benedito, Uberaba Minas Gerais, Brasil. e-mail:ma_ganshinho@hotmail.com

\section{Conflito de interesses}

Os autores declararam não haver conflito de interesses.

Processo de arbitragem

Recebido: 05/10/2017 Aprovado: 10/12/2017 


\section{Introdução}

Hoje em dia muitas evidências científicas ressaltam a importância da prática de atividades físicas e alimentação saudável, com isso as academias de ginásticas apresentam indivíduos que buscam estímulos e informações sobre como manter uma alimentação equilibrada aliada à práticadeexercíciosfísicos ${ }^{1,2}$.

Por sua vez, a mídia tem grande pressão na busca pelo corpo perfeito fazendo com que frequentadores de academias se deixem levar pela influência de amigos e dos próprios professores, porém muitas vezes essas pessoas não são preparadas adequadamente para dar orientações quanto ao consumo de suplementos alimentares já que médicos e nutricionistas são os únicos profissionais habilitados para indicar esses produtos ${ }^{3}$. Com isso é comum encontrar entre os frequentadores de academias aqueles que consomem suplementos sem conhecer realmente qual é a sua finalidade ${ }^{1}$.

Os suplementos alimentares são substâncias ingeridas pela via oral que tem como finalidade complementar determinada deficiência nutricional, porém na maioria das vezes eles são vendidos na forma de substâncias ergogênicas que servem para melhorar e aumentar o desempenho físico ${ }^{4,5}$ como reduzir gordura corporal, aumentar a massa muscular, estimular a recuperação e aumentar a capacidade aeróbica, atribuindo-Ihes também efeitos como perda de peso, prevenção de doenças, melhora da estética corporal e retardo dos efeitos adversos aoenvelhecimento ${ }^{6}$

Devido a esses efeitos, muitos praticantes de atividade física passam a consumir esses suplementos sem qualquer critério de indicação, acreditando no seu potencial milagroso e capaz de produzir resultados em curto prazo ${ }^{7}$.

De acordo com as normas brasileiras, os suplementos alimentares podem ser comercializados sob a forma de drágeas, tabletes, cápsulas, pós, granulados, pastilhas mastigáveis, líquidos, preparações semissólidas e suspensões ${ }^{8}$, sendo que esses suplementos se encontram divididos nas seguintes categorias: repositores hidroeletrolíticos, repositores energéticos, alimentos proteicos, alimentos compensadores, aminoácidos de cadeia ramificada e outros alimentos com fins específicos para praticantes de atividade física. Sendo que esses suplementos devem ser constituídos de um ou mais dos seguintes ingredientes: vitamina, mineral, erva ou outras plantas, aminoácidos, metabólitos, concentrados, constituintes e/ou extratos $^{9}$. Dentre essas categorias citadas os maisutilizados são os protéicos e energéticos ${ }^{10}$.

Segundo a portaria do Ministério da Saúde n-222 de 24 de março de 19988 essas categorias de suplementos são destinadas a praticantes de atividade física e estão dentro do subgrupo Alimentos para fins especiais, portanto não podem ser utilizados como alimentos convencionais ou como único item de uma dieta ${ }^{11}$.

Dentre os vários objetivos que podem fazer os frequentadores de academias consumirem suplementos alimentares, os mais citados são prevenção de doenças e o ganho de massa muscular'. Em estudo feito por Kirk e colaboradores $^{12}$ foi relatado que mulheres que faziam o uso de suplementos alimentares apresentavam um hábito de vida mais saudável com relação as não usuárias.

Vários fatores podem influenciar no conhecimento de uma pessoa como idade, sexo, nível de escolaridade e nível socioeconômico e dentre esses fatores o grau de escolaridade pode se interligar com algumas variáveis como raça, cultura, condição socioeconômica, comportamentos e fatores ambientais $^{13}$.

Sendo assim, este estudo tem como objetivo verificar a relação entre o grau de escolaridade e o uso de suplementos nutricionais por praticantes de atividade física em academias.

\section{Métodos}

Esta pesquisa tem característica prospectiva, transversal e está vinculada a um projeto maior "Uso de suplementos nutricionais em academias de Uberaba/MG" realizada no período de novembro de 2013 a abril de 2014 .

\section{Seleção das academias}

Os dados referentes ao número de academias atuantes em Uberaba e que fizeram parte deste estudo foram obtidos por meio de órgãos públicos e de informação: Lista telefônica de assinantes "lista SABE" e "guia SEI" e Conselho Regional de Educação Física de Minas Gerais. As listagens fornecidas por esses órgãos foram reunidas de forma a detectar coincidências dos nomes fantasia e jurídico das academias. Na vigência de coincidência de endereço, foi uniformizada apenas uma listagem de estabelecimentos. Por telefone, verificou-se a situação da academia quanto ao seu funcionamento atual e quais modalidades as mesmas ofereciam.

Os critérios de inclusão das academias foram: possuírem áreas físicas variadas e oferecerem diversas modalidades, sendo obrigatória pelo menos a musculação. Portanto, academias que só ofereciam um tipo de atividade (ex: só dança, pilates, lutas ou só natação) ou que as atividades fossem destinadas a somente uma faixa etária (ex: só para crianças ou só idosos) e gênero (ex: só mulheres ou homens) foram excluídas.

Após a definição de quais academias fariam parte do estudo, foi contatado por telefone o proprietário com o objetivo de se obter a permissão para a participação desse estabelecimento na pesquisa. Em casos onde ocorreu dificuldade de contato com os mesmos, e após 05 tentativas sem êxito, aquela instituição foi excluída. Após o primeiro contato, por telefone, com o proprietário da academia e sua concordância em ouvir sobre o projeto, o estabelecimento foi visitado para que o estudo fosse apresentado com detalhes e se obtivesse a autorização por meio da assinatura da carta de anuência padrão. Em seguida foi realizado o preenchimento dos dados referentes ao número de indivíduos matriculados, profissionais atuantes, presença do comércio de suplementos e modalidades oferecidas pela academia na ficha de identificação padronizada. Esta ficha permitiu o levantamento do número de matriculados para que fosse realizado, posteriormente, o cálculo amostral do presente estudo.

Seguindo os critérios acima, foram catalogadas 19 academias, porém fizeram parte deste estudo 15 delas, pois em uma não foi possível o contato com o responsável, após todas as tentativas, e três se recusaram a participar do estudo.

\section{Instrumento de coleta de dados e aplicação}

O instrumento utilizado para coleta dos dados foi um questionário autoaplicável e padronizado de múltipla escolha com questões pertinentes ao assunto e que foi previamente testado por seus autores, para correções, antes de ser utilizado para fim científico.

Estudantes de graduação em nutrição previamente treinados fizeram a coleta de dados, sem necessidade de identificação do avaliado; coube a eles apenas verificar cada questionário após o preenchimento, antes da liberação do voluntário, de forma a evitar dados incompletos ou inadequadamente respondidos.

\section{Seleção e descrição da amostra}

Foram incluídos no estudo somente indivíduos com idade $\geq 18$ anos que estavam matriculados nas academias e, portanto, fossem fisicamente ativos (que praticassem alguma modalidade pelo menos duas vezes por semana).

O cálculo amostral realizado com base na seguinte premissa: considerando-se que não há dados de estudos similares realizados na cidade de Uberaba, a amostra foi calculada por meio da equação $n=p .(1-p) . z 2 / e 2$ em que $p$ foi a proporção estimada a partir de estudos no Brasil de indivíduos que usam suplementos (50\%), z2 o escore padrão da distribuição normal (definido como 1,96 para uma pesquisa com 95\% de confiança) e e2 a margem de erro do estudo (3\%)

O número de indivíduos por academia foi calculado com base no número total de praticantes em cada uma e a porcentagem que estes representaram na somatória geral de todos os estabelecimentos.

Os pesquisadores abordaram de forma aleatória, na entrada principal da academia, em diferentes horários do dia (diurno e noturno) e da semana, os potenciais participantes do estudo. Estes foram informados sobre o objetivo da pesquisa, da não necessidade de sua identificação para responder o questionário e, da garantia de anonimato de suas informações e, só foram aceitos no estudo, após assinarem o termo de consentimento 
livre e esclarecido - TCLE, de forma que pudessem desistir a qualquer momento, sem que isso lhes oferecesse prejuízo.

\section{Caracterização da escolaridade}

Para classificar os indivíduos quanto a sua escolaridade, os mesmos responderam no questionário uma pergunta referente ao nível de escolaridade e a partir disso foram classificados em dois grupos, baixa escolaridade (até $2^{\circ}$ grau completo - ensino médio completo) e alta escolaridade (a partir da entrada na graduação com ou sem pós-graduação).

\section{Aspectos éticos}

O estudo foi submetido e aprovado pelo Comitê de Ética em Pesquisa da Universidade Federal do Triângulo Mineiro (UFTM) sob o protocolo 2490 .

\section{Análise estatística}

Além da análise descritiva, foi utilizado o teste Qui-quadrado para verificar a associação entre variáveis categóricas quanto ao uso de suplementos alimentares de acordo com a escolaridade. Para isso utilizou-se o Software Statistical Package for the Social Sciences (SPSS) versão 17 e $p$-Valor $<0,05$

\section{Resultados}

Participaram do estudo 660 frequentadores de academias de ginástica da cidade de Uberaba (MG), com idade entre 18 e 84 anos, de ambos os sexos, sendo a maioria homens (58,9\%).

Os participantes caracterizaram-se por ser a maioria do sexo masculino $389(58,9 \%)$, sendo que a maior parte dos homens $270(40,9 \%)$ apresenta alta escolaridade, assim como a maior parte das $208(31,5 \%)$.

A prevalência do consumo de suplementos entre os frequentadores de academia foi de 317 indivíduos $(48,0 \%)$, sendo os indivíduos com alta escolaridade os responsáveis pelo maior consumo de suplementos alimentares 234 (35,5\%). Porém a maior parte dos indivíduos refere não utilizar suplementos alimentares 343 (52\%).

A tabela 1 apresenta a prevalência e/ ou os tipos de suplementos alimentares consumidos por indivíduos com alta e baixa escolaridade.

Tabela 1

Tipos de suplementos alimentares consumidos por praticantes de atividade física de acordo com o nível de escolaridade.

\begin{tabular}{|c|c|c|c|c|}
\hline $\begin{array}{c}\text { Quais } \\
\text { Suplementos } \\
\text { usam }\end{array}$ & $\begin{array}{l}\text { Geral } \\
\mathrm{N}(317) \\
\mathrm{n}(\%)\end{array}$ & $\begin{array}{c}\text { Alta } \\
\text { Escolaridade } \\
\mathrm{N}(234) \\
\mathrm{n}(\%) \\
\end{array}$ & $\begin{array}{c}\text { Baixa } \\
\text { Escolaridade } \\
\mathrm{N}(83) \\
\mathrm{n}(\%) \\
\end{array}$ & $\begin{array}{c}\mathrm{p}- \\
\text { Valor } \\
\star\end{array}$ \\
\hline $\begin{array}{c}\text { Usa mais de um } \\
\text { tipo de } \\
\text { suplemento }\end{array}$ & $206(65,0)$ & $161(50,7)$ & $45(14,3)$ & \\
\hline $\begin{array}{l}\text { Ricos em } \\
\text { Proteína }\end{array}$ & $53(16,7)$ & $35(11)$ & $18(5,6)$ & \\
\hline $\begin{array}{l}\text { Anabolizantes } \\
\text { com ou sem } \\
\text { outros }\end{array}$ & $12(3,7)$ & $9(2,8)$ & $3(0,9)$ & \\
\hline BCAA & $11(3,5)$ & $7(2,2)$ & $4(1,2)$ & \\
\hline $\begin{array}{l}\text { Vitaminas e } \\
\text { Minerais }\end{array}$ & $10(3,2)$ & $7(2,2)$ & $3(0,9)$ & \\
\hline $\begin{array}{c}\text { Queimadores de } \\
\text { Gordura }\end{array}$ & $06(1,9)$ & $3(0,9)$ & $3(0,9)$ & \\
\hline $\begin{array}{l}\text { Bebidas } \\
\text { Isotônicas }\end{array}$ & $05(1,6)$ & $3(0,9)$ & $2(0,6)$ & 0,17 \\
\hline $\begin{array}{l}\text { Hipercalóricos ou } \\
\text { compensadores }\end{array}$ & $04(1,3)$ & $1(0,3)$ & $3(0,9)$ & \\
\hline $\begin{array}{l}\text { Ricos em } \\
\text { Carboidrato }\end{array}$ & $03(0,9)$ & $3(0,9)$ & 0,0 & \\
\hline $\begin{array}{l}\text { Shakes } \\
\text { Creatina }\end{array}$ & $\begin{array}{l}02(0,6) \\
01(0,3)\end{array}$ & $\begin{array}{c}2(0,6) \\
0,0\end{array}$ & $\begin{array}{c}0,0 \\
1(0,3)\end{array}$ & \\
\hline $\begin{array}{c}\text { Outros } \\
\text { Aminoácidos }\end{array}$ & $01(0,3)$ & 0,0 & $1(0,3)$ & \\
\hline $\begin{array}{c}\text { Naturais e } \\
\text { Fitoterápicos }\end{array}$ & $01(0,3)$ & $1(0,3)$ & 0,0 & \\
\hline Outros & $01(0,3)$ & $1(0,3)$ & 0,0 & \\
\hline
\end{tabular}

Teste Qui-quadrado ( $\mathrm{p}$-Valor $<0,05)$

Os indivíduos com alta escolaridade consomem preferencialmente mais de um tipo de suplemento $161(50,7 \%)$, sendo os ricos em proteínas $35(11 \%)$, anabolizantes $9(2,8)$ os mais consumidos. Já entre os indivíduos com baixa escolaridade se destacam entre os mais consumidos, os ricos em proteínas $18(5,6 \%)$ e BCAA $4(1,2 \%)$ (figura 1$)$.

Não houve diferença estatística entre a concepção sobre a própria alimentação entre os diferentes níveis de escolaridade, sendo que em sua maioria 485 (73,5\%) ambos consideram ter uma boa alimentação, a qual pode ser melhorada. Com relação ao tempo em que já pratica atividade física, a maioria 374 $(56,7 \%)$ frequenta a academia a mais de um ano, com uma frequência de três a cinco vezes na semana 459 (69,5\%) em ambos os níveis de escolaridade, sendo a musculação associada à algum outro tipo de atividade esportiva as mais praticadas pelos frequentadores das academias 391 (59,2\%).

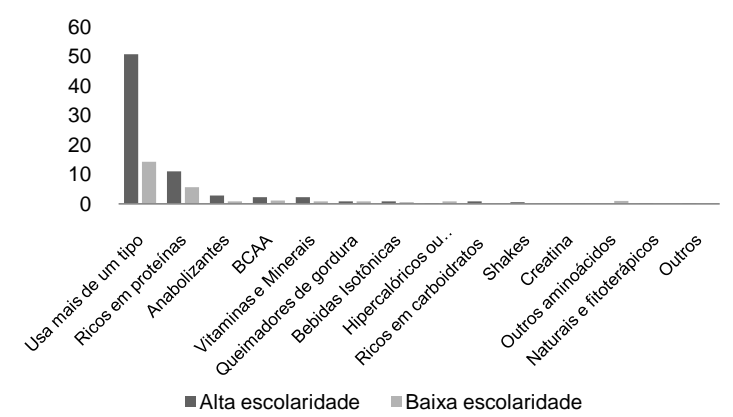

Figura1. Nível de escolaridade e consumo de suplementos alimentares

Comparações sobre a quantidade de suplementos alimentares utilizadas, os objetivos e se obteve os resultados esperados entre os diferentes níveis de escolaridade são apresentadas na Tabela 2.

Não há diferença estatística quanto ao uso de suplementos alimentares entre os indivíduos com alta e baixa escolaridade. Entretanto, entre os que utilizam, os com alta escolaridade em sua maioria $61(9,2 \%)$ fazem uso de 2 suplementos, enquanto os com baixa escolaridade em sua maioria 66 (10\%), utilizam apenas 1 tipo de suplemento. O principal objetivo de utilizar suplementos é o mesmo nos dois grupos, sendo mais de um motivo e entre esses motivos os principais são ganho de força, massa muscular e perda de peso. As pessoas com alta escolaridade que referiram fazer uso de suplementos relatam ter conseguido o resultado desejado 130 (19,6\%), no entanto as pessoas com baixa escolaridade estavam divididas entre, já obtiveram resultado desejado $34(5,2 \%)$ e ainda não sabiam se obtiveram o resultado, $39(5,9 \%)$.

Com relação à fonte de indicação para 0 uso de suplementos, a maioria não teve sua indicação por nutricionista, $249(78,5 \%)$, e sim por outras pessoas não habilitadas, como amigos, vendedor da loja de suplementos, personaltrainer, indicação própria. Isso ocorreu tanto em indivíduos com alta escolaridade 182 (57,4\%), como em indivíduos com baixa escolaridade $67(21,1 \%)$.

\section{Discussão}

Diante do exposto no presente estudoverifica-se que entre os frequentadores das academias a maioria 478 (72,4\%) apresenta alta escolaridade (cursando ou que já cursaram o ensino superior), resultado semelhante ao encontrado no estudo de Santos e Santos ${ }^{14}$ onde do total de pessoas que participaram do estudo $(76 \%)$ estavam cursando o $3^{\circ}$ grau (ensino superior) e no de Frizon e colaboradores ${ }^{15}$ onde $62,6 \%$ estavam cursando ou já concluíram o nível superior. Sendo no presente estudo demonstrado um expressivo consumo de suplementos alimentares entre os frequentadores das academias, principalmente entre os homens. Outros estudos também demonstram consumo significativo de suplementos alimentares ${ }^{3,16,17,18}$, principalmente entre os homens ${ }^{16}$.

Não houve diferença estatística quanto ao uso de suplementos alimentares entre os indivíduos com alta e baixa escolaridade, porém algumas características são observadas em cada grupo, lembrando que a maioria das pessoas não faz uso de suplementos $345(52,3 \%)$. 
Tabela 2

Uso de suplementos alimentares relacionados ao nível de escolaridade.

\begin{tabular}{|c|c|c|c|c|}
\hline \multirow[b]{2}{*}{ Suplementos } & \multicolumn{4}{|c|}{ Nível de escolaridade } \\
\hline & $\begin{array}{c}\text { Geral }(\mathrm{N}=660) \\
\mathrm{n}(\%)\end{array}$ & $\begin{array}{c}\text { Alta escolaridade } \\
(\mathrm{N}=274) \\
\mathrm{n}(\%)\end{array}$ & $\begin{array}{c}\text { Baixa escolaridade } \\
(\mathrm{N}=386) \\
\mathrm{n}(\%)\end{array}$ & p-Valor \\
\hline \multicolumn{5}{|l|}{ Quantos suplementos utiliza } \\
\hline 1 suplemento & $101(15,3)$ & $35(5,3)$ & $66(10)$ & \multirow{5}{*}{0,10} \\
\hline 2 suplemento & $74(11,1)$ & $61(9,2)$ & $13(1,9)$ & \\
\hline 3 suplemento & $65(9,9)$ & $47(7,1)$ & $18(2,7)$ & \\
\hline Mais de 3 suplemento & $77(11,6)$ & $60(9)$ & $17(2,6)$ & \\
\hline Não faz uso & $345(52,3)$ & $246(37,7)$ & $99(15)$ & \\
\hline \multicolumn{5}{|l|}{ Qual o objetivo de utilizar } \\
\hline Perder peso & $23(3,4)$ & $13(1,9)$ & $10(1,5)$ & \multirow{10}{*}{0,08} \\
\hline Repor nutrientes, energia e evitar fraqueza & $13(2)$ & $9(1,3)$ & $4(0,6)$ & \\
\hline Reduzir o estresse, sentir-se melhor, mais disposto & $02(0,3)$ & 0,0 & $2(0,3)$ & \\
\hline Substituir refeições & 0 & 0,0 & 0,0 & \\
\hline Ganho de massa muscular, forca & $82(12,4)$ & $56(8,4)$ & $26(3,9)$ & \\
\hline Para suprir deficiências alimentares & $10(1,5)$ & $5(0,7)$ & $5(0,7)$ & \\
\hline Melhorar desempenho & $03(0,4)$ & $2(0,3)$ & $1(0,1)$ & \\
\hline Não faz uso & $345(52,3)$ & $132(20)$ & $213(32,3)$ & \\
\hline Vários (mais de um motivo) & $145(21,9)$ & $114(17,2)$ & $31(4,6)$ & \\
\hline Perder peso e ganhar massa, força (com ou sem outros) & $32(4,8)$ & $28(4,2)$ & $4(0,6)$ & \\
\hline \multicolumn{5}{|l|}{ Obteve o resultado desejado } \\
\hline Sim & $164(24,8)$ & $130(19,6)$ & $34(5,2)$ & \multirow{4}{*}{0,88} \\
\hline Não & $20(3)$ & $11(1,6)$ & $9(1,4)$ & \\
\hline Ainda não sei & $130(19,6)$ & $91(13,7)$ & $39(5,9)$ & \\
\hline Não faz uso & $343(51,9)$ & $244(36,9)$ & $99(15)$ & \\
\hline
\end{tabular}

Teste Qui-quadrado (p-Valor<0,05)

No estudo de Pereira e colaboradores ${ }^{1}$ foram entrevistadas 309 pessoas em uma academia com a maioria (69,9\%) apresentando nível superior (completo ou não) onde observaram que $23,9 \%$ faziam uso de algum tipo de suplemento alimentar. Diferente desses achados, de acordo com Moreira et al. ${ }^{19}$, foram entrevistado 60 pessoas em uma academia com idade média de $23,28( \pm 3,37)$ anos onde observaram que $31,7 \%$ faziam uso de algum tipo de suplemento alimentar.Sendo assim, a prevalência de uso, varia conforme as características do estudo.

Com relação ao tempo que as pessoas com alta e baixa escolaridade praticam atividade física, destacou-se no presente estudo que a maioria frequenta a academia a mais de um ano, de três a cinco vezes na semana treinando de uma a duas horas por dia, sendo a musculação associada a mais de uma categoria (por exemplo: atividades aeróbicas e aquáticas) as mais praticadas, $59,2 \%$ dos participantes. Esses achados corroboram com outros estudos como de Hallack e colaboradores ${ }^{25}$, que avaliou uso de suplementos em academias e destacaram a musculação como a atividade física mais realizada (72\%), enquanto Duarte e colaboradores ${ }^{20}$ observaram que $64,3 \%$ dos praticantes, associam musculação e algum exercício aeróbio, evidenciando que a musculação combinada ou não a outras atividades é a mais praticada nas academias.

No presente estudo a maior parte dos indivíduos relata frequentar a academia regularmente há mais de um ano, o que demonstra que com a prática prolongada, a tendência é buscar suplementos para reforçar os resultados do exercício. O tempo de pratica de exercícios e a frequência à academia afetam o usode suplemento, pois o individuo é mais exposto ao ambiente de consumo, segundo Hirschbruch; Fisberg; Mochizuki ${ }^{16}$

A concepção quanto à alimentação referida por ambos os grupos tanto de alta quanto de baixa escolaridade do presente estudo é considerada boa, mas pode melhorar. Segundo o estudo de Moreira e colaboradores ${ }^{19}$ em que avaliaram o uso de suplementos alimentares, consumo alimentar e conhecimento nutricional dos praticantes de exercícios físicos, concluíram que a alimentação dos praticantes de exercício não se mostrou adequada do ponto de vista nutricional, demonstrando quea falta de informações confiáveis em relação à nutrição pode levar à manutenção de hábitos alimentares inadequados ou consumo errôneo de suplementos alimentares, o que pode acarretar prejuízos na prática de exercícios físicos ${ }^{21}$.

Com relação ao tipo de suplemento mais consumido se destacam os ricos em proteínas para ambos os níveis de escolaridade, seguido dos anabolizantes para os indivíduos com alta escolaridade e dos aminoácidos de cadeia ramificada (BCAA) para as pessoas com baixa escolaridade. Lembrando que os anabolizantes não são considerados suplementos alimentares, porém é um item utilizado no questionário e que merece ser considerado tendo em vista o consumo prevalente entre praticantes de atividade física em academias ${ }^{15,18,22}$ principalmente entre praticantes de musculação ${ }^{15,23,24}$.Para Santos e Santos ${ }^{14}$ aminoácidos e creatina são preferencialmente utilizados por alunos que possuem $01^{\circ}$ e $2^{\circ}$ graus, enquantovitaminas, sais minerais, compostos para emagrecimento, creatina e L-carnitina são preferencialmente utilizados por alunos do $3^{\circ}$ grau. Gomes e colaboradores ${ }^{10}$ relatam que os suplementos mais consumidos por praticantes de atividade física são os aminoácidos de cadeia ramificada, ricos em proteína e maltodextrina. Já Fayh e colaboradores ${ }^{3}$ relatam maior consumo de suplementos proteicos, aminoácidos de cadeia ramificada e suplementos calóricos. Hallak; Fabrini e Peluzio $^{25}$ destacaram uso de aminoácidos ou concentrados proteicos $(37,1 \%)$ seguidos de creatina $(28,6 \%)$ e bebidas carboidratadas $(12,8 \%)$. Moreira e Rodrigues ${ }^{19}$ afirmam que os suplementos mais consumidos são os aminoácidos ou concentrados proteicos $(48,28 \%)$, seguido por suplementos ricos em carboidratos $(34,48 \%)$, vitaminas ou complexos vitamínicos $(17,24 \%)$.

Dentre os vários motivos (objetivos) que levam as pessoas com alto e baixo nível de escolaridade a utilizarem suplementos alimentares, a maioria relatou fazer o uso por mais de um motivo $(21,9 \%)$ entre eles os principais são ganho de força e massa muscular $(12,4 \%)$, perda de peso $(3,4 \%)$ e os dois juntos (perda de peso e ganho de força e massa muscular) $(4,8 \%)$. O mesmo resultado vai ao encontro de outros estudos ${ }^{1,17,16}$ no qual o objetivo de todosfoi avaliar o uso de suplementos. No estudo de Santos e colaboradore ${ }^{27}$ os fatores motivacionais mencionados nas pesquisas foram as possíveis melhorias no treinamento e alterações estéticas. Estes dados mostram uma tendência da população pela busca incessante do corpo esteticamente perfeito $^{27}$ e demonstram que as pessoas buscam os exercícios e os suplementos como uma forma de modificar o peso, seja para ganhar ou perder ${ }^{16}$

Ainda quanto ao uso de suplementos, as pessoas com alta escolaridade relataram ter conseguido o resultado desejado, já as pessoas com baixa escolaridade, ainda não sabem se obtiveram resultado ou não. No estudo de Alves e colaboradores $^{28}$, verificaram que todas as pessoas alcançaram o resultado almejado. Já no estudo de Brito e colaboradores ${ }^{29}$, foi verificado que $80 \%$ das pessoas que utilizaram suplementosconseguiram o resultado. Porém estudo como o de Schneider e colaboradores ${ }^{30}$ mostra que os entrevistados que utilizam suplementos nutricionais pratica exercícios físicos há mais de um ano, demonstrando de acordo com o estudo de Fontes e Navarro ${ }^{31}$ a importância de manter uma rotina de exercício regular para potencializar ou obter resultados com a suplementação. 
Com relação à fonte de indicação para 0 uso de suplementos, nutricionistas e médicos, os quais são os profissionais habilitados para tal, não foram as fontes mais citadas, sendo amigos, vendedor da loja de suplementos, personaltrainer e indicação própria os mais citados $(78,5 \%)$. Assim como no estudo de Fayh e colaboradores ${ }^{3}$ onde observaram que $38,6 \%$ consumiram suplementos por indicação de educador físico, 9,8\% por nutricionistas e $51,6 \%$ por outros (amigos, vendedores de lojas e outros profissionais da saúde), isto pode estarrelacionado com o fato de que muitas lojas de suplementos estão localizadas dentro de academias de ginástica, fazendo com que as pessoas que circulam pelo ambiente estejam mais expostas a este tipo de apelo comercial. Para Santos e Santos ${ }^{14}$ aqueles que possuem 1 e $2^{\circ}$ graus adquirem os seusprodutos em lojas especializadas, já os alunos com $3^{\circ}$ grau compram os suplementos na academia,com o professor ou em farmácias, portanto independente do nível de escolaridade observa-seuma tendência de ocorrer incentivo interalunos,assim como, entre professor e aluno para usar osuplemento alimentar visando melhorar o desempenho. Vale lembrar também que a falta de informações confiáveis em relação à nutrição pode levar à manutenção de hábitos alimentares inadequados ou consumo errôneo de suplementos alimentares, o que pode acarretar prejuízos na prática de exercícios físicos ${ }^{21}$

\section{Conclusão}

O nível de escolaridade não interfere no uso de suplementos alimentares por praticantes de atividade física em academias. Porém observou-se prevalência significativa no consumo tanto entre os indivíduos com alta como em baixa escolaridade, sendo os ricos em proteínas os mais consumidos em ambos os grupos.

Vale ressaltar que há grande influência de pessoas não habilitados para a indicação do uso de suplementos, o que muitas vezes acarreta no uso errôneo desses produtos.

\section{Referências}

1. Pereira RF, Lajolo FM, Hirschbruch MD. Consumo de suplementos por alunos de academias de ginástica em São Paulo. Rev Nutr. 2003. 16(3): 265-272.

2. Antunes, AC. Perfil profissional de instrutores de academias de ginástica e musculação. Ver Dig. 2003. 9(60): 132-140.

3. Fayh et al. Consumo de suplementos nutricionais por frequentadores de academias da cidade de Porto Alegre. Rev Bras Ciênc Esporte. 2013. 35(1): 27-37.

4. Alves C, Lima RVB. Uso de suplementos alimentares por adolescentes. J Pediatr. 2009. 85(4): 287-294.

5. Bucci LR. Select herbal sand human exercise performance. Am J Clin Nutr. 2002. 72(2): 624S-636S.

6. Ortega J O. Sports nutrition. Int J Athletic Therapy Training. 2004. 9(5): 6870.

7. Williams MH. Dietary supplements and sports performance: aminoacids. J Int Society of Sports Nutr.2005. 2(2): 63-67.

8. Ministério da Saúde. Secretaria da Vigilância Sanitária. Portaria n.222 de 24 de março de 1998. Regulamento técnico para fixação de identidade e características mínimas de qualidade que deverão obedecer os alimentos para praticantes de atividade física. Diário Oficial da União de 24 de março de 1998. Brasília. 1998.

9. Morrison LJ,Gizis F, Shorter B. Prevalent use of dietary supplements among people who exercise at a commercial gym. Int $\mathrm{J}$ of Sport Nutrition \& Exercise Metabolism. 2004. 14(4): 481-492.

10. Gomes GS, Degiovanni GC, Garlipp MR, Chiarello PG, Jordão AA Caracterização do consumo de suplementos nutricionais em praticantes de atividade física em academias. Medicina (Ribeirão Preto). 2008. 41(3): 327-331.

11. Melethil S. Proposed rule: current good manufacturing practice in manufacturing, packing, or holding dietary ingredients and dietary supplements. Life Science. 2006. 78(18); 2049-2053.

12. KirkSFL.et al. Diet and lifestyle characteristics associated with dietary supplement use in women. Public Health Nutrition, Wallingford 1998. 2(1) 69-73.

13. Alves ICB.Variáveis significativas na avaliação da inteligência. Psicol Esc Educ. 1998. 2(2): 109-114.

14. Santos RP, Santos MAA. Uso de suplementos alimentares como forma de melhorar a performance nos programas de atividade física em academias de ginástica. Rev Paul Educ Fís. 2002. 16(2): 174-185

15. Frizon F, Macedo SMD. Uso de esteróides andrógenos anabólicos por praticantes de atividade física das principais academias de Erechim e Passo Fundo/RS. Rev Ciênc Farmacêutica Básica e Aplicada. 2005. 26(3): 227-232.

16. Hirschbruch MD, Fisberg M, Mochizuki L. Consumo de Suplementos por Jovens Frequentadores de Academias de Ginástica em São Paulo. Rev Bras Med Esporte. 2008. 14(6): 265-272.

17. Araújo ACM, Soares $Y$ NG. Perfil de utilização de repositores proteicos nas academias de Belém, Para. Rev Nutr Camp. 12(8): 1-9.

18. Araújo LR, Andreolo J, Silva MS. Utilização de suplemento alimentar e anabolizantes por praticantes de musculação nas academias de GoiâniaGO. Rev Bras Ciênc Mov. 10(3): 13-18.

19. Moreira FP, Rodrigues KL. Conhecimento nutricional e suplementacãa alimentar por praticantes de exercício físico. Rev Bras Med Esp. 2014 2(5): 370-373.

20. Duarte PSF et al. Aspectos gerais e indicadores estatísticos sobre o consumo de suplementos nutricionais em academias de ginástica. Rev Nutr Pauta. 2007. p. 27-31.

21. Duran ACFL, Latorre MRDO, Florindo A, Jaime PC. Correlação entre consumo alimentar e nível de atividade física habitual de praticantes de exercícios físicos em academia. Rev Bras Ciênc Mov 2004 12(1): 15-29.

22. Silva LSMF, Moreau RLM. Uso de esteróides anabólicos androgênicos por praticantes de musculação de grandes academias da cidade de São Paulo. Rev Bras Ciênc Farmacia. 2003. 29(3): 27-32.

23. Iriart JAB, Chaves JC, Orleans RG. Culto ao corpo e uso de anabolizantes entre praticantes de musculação. Caderno de Saúde Pública. 2009. 25(4): 773-782.

24. Cerqueira, G. S. et al. Perfil do consumo de anabolizantes em praticantes de atividade física da cidade de João Pessoa. Rev Dig. 2010. 15(147).

25. Hallak A, Francini S, Peluzio MCG. Avaliação do consumo de suplementos nutricionais em academias da zona sul de Belo Horizonte - Minas Gerais. Rev Bras Nutr Esport. 2007. 1(2): 55-60.

26. Fleischer B, Read M. Food supplement usage by adolescent males. Adolesc. 1982. 17(8): 31-45.

27. Santos HVD, Oliveira CCP, Freitas AKC, Navarro AC. Consumo de suplementos alimentares por praticantes de exercício físico em academias de bairros nobres da cidade do Recife. Rev Bras Nutr Esport. 2013. 7(40): 204-211.

28. Alves SCR, Navarro F. O uso de suplementos alimentares por frequentadores de academias de Potim-SP. Rev Bras Nutr Esport. 2010. 4(20): 139-146.

29. Brito DS, Liberali R Perfil do consumo de suplemento nutricional por praticantes de exercício físico nas academias da cidade de Vitória da Conquista-BA. Rev Bras Nutr Esport. 6(31): 66-75.

30. Schneider C, Machado C, Laska SM, Liberali R. Consumo de suplementos nutricionais por praticantes de exercício físico em academias de musculação de Balneário Camboriú - SC. Rev Brasil Nutr Esport. 2008. 2(11): 307-322.

31. Fontes, A. M. S. A., Navarro, F. Consumo de suplementos nutricionais por praticantes de atividades físicas em academias de Sete Lagoas - MG. Rev Bras Nutr Esportiva. 2010. 4(24): 515-523. 